\title{
Myasthenia gravis as a prognostic marker in patients with thymoma
}

\section{Bernardo Cacho-Díaz ${ }^{1}$, Karen Salmerón-Moreno ${ }^{1}$, Nydia A. Lorenzana-Mendoza ${ }^{1}$, Julia Texcocano ${ }^{1}$, Oscar Arrieta $^{2}$}

${ }^{1}$ Neuroscience Unit, Instituto Nacional de Cancerología, México City, México; ${ }^{2}$ Thoracic Oncology Unit, Instituto Nacional de Cancerología, México City, México

Contributions: (I) Conception and design: B Cacho-Díaz, O Arrieta; (II) Administrative support: B Cacho-Díaz, O Arrieta; (III) Provision of study materials or patients: K Salmerón-Moreno, B Cacho-Díaz; (IV) Collection and assembly of data: NA Lorenzana-Mendoza, B Cacho-Díaz; (V) Data analysis and interpretation: J Texcocano, O Arrieta; (VI) Manuscript writing: All authors; (VII) Final approval of manuscript: All authors.

Correspondence to: Bernardo Cacho-Díaz. Av. San Fernando 22, Sección XVI Tlalpan Z.C. 14080 México City, México.

Email: bernardocacho@doctor.com.

\begin{abstract}
Background: Thymoma is the most common mediastinal tumor, representing $<1 \%$ of all cancers. It is usually associated with paraneoplastic disorders, mainly myasthenia gravis (MG). The aim of the present study was to describe patients with thymoma and the differences between those with MG and those without it.

Methods: A retrospective 10-year database of the patients with thymoma treated at a single cancer referral hospital (National Institute of Cancer, Mexico City), was analyzed.

Results: Sixty-four files from patients with thymoma were analyzed, 18 of them had MG. The symptoms that occurred most frequently in patients with MG were ptosis, diplopia, appendicular weakness, dysphonia and dysphagia. The most frequent Myasthenia Gravis Foundation of America (MGFA) stage was IIIb followed by stage I. Almost all the patients with MG had positive Acetylcholine Receptor antibodies $(\mathrm{P}<0.001)$, with not specified antibodies in four patients. The median overall survival showed a trend to be higher among the patients with MG, but there were no significant differences.

Conclusions: In patients with thymoma MG manifests with different clinical and autoimmune traits, but not survival differences. A larger multi-centric study should be encouraged to evaluate the prognostic implications of having MG in patients with thymoma.
\end{abstract}

Keywords: Thymoma; myasthenia gravis (MG); acetylcholine receptor (AChR)

Submitted Dec 16, 2017. Accepted for publication Mar 29, 2018.

doi: $10.21037 /$ jtd.2018.04.95

View this article at: http://dx.doi.org/10.21037/jtd.2018.04.95

\section{Introduction}

Thymic epithelial tumors (TET) are mainly composed of thymomas $(\mathrm{T})$ and thymic carcinomas; they are the most common primary neoplasms of the anterior mediastinum. Thymomas are the most frequent mediastinal neoplasm, their frequency is about $20 \%$ of cases $(1,2)$. Thymic carcinomas are more aggressive neoplasms and their relation with autoimmune diseases is extremely rare, due to its cytological atypia and lack of thymus like features (2). Compared with all cancers, thymomas account for less than
$0.5 \%$, with an incidence of 0.15 per 100,000 person years (3). The mean age at the time of diagnosis is 52 years, without gender predominance. TET are usually asymptomatic and are found as an incidental mediastinal mass in chest $\mathrm{X}$-ray. One-third of the patients with TET have chest pain, cough or dyspnea related to tumor compression or invasion (4).

Myasthenia gravis (MG) is a rare neuromuscular disease caused by antibodies against neuromuscular junction and represents the most common paraneoplastic disease associated with thymoma (5), however, a wide range of autoimmune diseases have been reported, i.e., Cushing 
syndrome, pure red blood cell aplasia, rheumatoid arthritis, immunodeficiency (Good's syndrome) and thymomaassociated multiorgan autoimmunity (6,7). Most patients with MG associated thymoma have antibodies against the postsynaptic nicotinic acetylcholine receptor (AChR) (8). Nevertheless, non-AChR muscle antibodies could also be present in more than $95 \%$ of thymoma patients, targeted mainly against striated muscle titin and ryanodine receptor (strational antibodies), and are usually associated with a severe and late-onset form of MG $(9,10)$.

MG is present in $30-50 \%$ of patients with thymoma, while $10-20 \%$ of patients with MG have thymoma (although some report an association as high as $80 \%)(2,5,8)$. Thymoma associated MG is considered to have a worse prognosis and poor outcome, being thymoma a negative prognostic factor between MG patients; paradoxically, some have postulated that patients with thymoma and MG have a survival advantage over patients without MG; thus a prognostic significance has not yet been established (11-13).

Thymomas are classified based upon their histologic appearance in five subtypes according to the World Health Organization (WHO) system: A, AB, B1, B2, B3, and thymic carcinoma cases are classified as C. Most of the data supporting treatment decisions are based on the most widely used tool for staging thymic malignancies: the Masaoka-Koga staging system (14).

Thymectomy is the cornerstone treatment modality in patients with early stages of TET and is an approved treatment modality for patients with seropositive MG even without thymoma (15). Thymectomy in patients with $\mathrm{T}$ has a probability of complete remission of $28-48 \%$ (16), a complication rate of $20 \%$ (blood vessel damage, postoperative myasthenic crisis, pain, and infection) and a $2 \%$ mortality (17).

The present study describes the clinical features and prognosis of the patients with TET and MG and compares them to those without MG.

\section{Methods}

At a cancer referral hospital (National Institute of Cancer, Mexico City), all the files of the patients with TET from 2006-2016 were analyzed. The database collected: demographic, clinical symptoms, oncologic history, comorbid paraneoplastic disorders, and outcome characteristics. Patients with TET and myasthenia gravis (TMG) were compared to those without myasthenia gravis (TnMG). The study was reviewed and approved by our
Institutional ethics and scientific committees (INCAN/ CI/837/17). Variables were compared using the chi-square or Student T tests according to the case. Prognosis was analyzed using Kaplan-Meier curves with log-rank test to identify the differences between the groups, $\mathrm{P}<0.05$ was considered to be statistically significant. SPPS Statistics v.18 software (IBM Corp., Armonk, NY, USA) was used to perform the analysis.

\section{Results}

We report 64 patients with thymoma, 35 (54.7\%) were men, $29(45.3 \%)$ were women; the most common WHO stage was B (28\%). Eighteen patients (28.1\%) were diagnosed with MG as seen in Table 1. We classified MG patients according to the Myasthenia Gravis Foundation of America (MGFA), and the most common stage was IIIb followed by stage I (Table 2). The mean age at the time of thymoma diagnosis was 51.4 years (range, $16-81$ years) and the mean age at the time of thymectomy was 52.5 years; the mean age at the time of MG diagnosis was 49.6 years (range, 27-65 years). The mean latency of time between the diagnosis of MG and thymectomy was 8 months. All patients had biopsy proven TET; according to the treatment, 34 (53.1\%) patients underwent surgery, of which, $30(88.2 \%)$ were treated with extended transsternal thymectomy; and $3(8.8 \%)$ were treated with partial median sternotomy thymectomy, two of them due to non resectable tumor and the last one due to hemodynamic complications during surgery. The detailed first line therapy is described in Table 3.

After a mean of 3-year follow-up three TMG patients died, causes of death were due to postoperative cardiac arrest and Good's Syndrome. In the TnMG, ten patients died: five deaths were due to cardiac arrest, four due to cancer progression and one due to hypovolemic shock. Figure 1 and Table 4 shows the overall survival and the survival differences between both groups; no difference in overall survival was seen between patients with thymoma or thymic carcinoma. Clinical findings, metastases and acetylcholine receptor antibody (AChr-Ab) comparing TMG and TnMG groups, are described in Table 5. Most frequent symptoms in TMG were: diplopia 15 (83\%), appendicular weakness 13 (72\%), ptosis 13 (72\%), dysphagia $11(61 \%)$ and speech impairment $9(50 \%)$, the rest of the symptoms were not statistically significant. Metastatic disease was found more frequent in TnMG (8 patients had lung, 7 bone, 4 hepatic and 1 CNS metastases). AChR-Ab was positive in 14 (78\%) MG patients, and not specified in 
Table 1 Characteristics of thymoma patients with and without myasthenia gravis

\begin{tabular}{|c|c|c|c|c|}
\hline Characteristic & All $(n=64), \%(n / N)$ & \multicolumn{2}{|c|}{ Thymoma } & $\mathrm{P}$ \\
\hline Gender & & & & 0.930 \\
\hline Female & $45.3(29 / 64)$ & $44.4(8 / 18)$ & $45.7(21 / 46)$ & \\
\hline Male & $54.7(35 / 64)$ & $55.6(10 / 18)$ & $54.3(25 / 46)$ & \\
\hline Mean $( \pm S D)$ & $51.4(13.0)$ & $50.8(10.7)$ & $51.7(13.8)$ & 0.966 \\
\hline$<50$ & $43.7(28 / 64)$ & $38.9(7 / 18)$ & $45.7(21 / 46)$ & \\
\hline$>50$ & $56.3(36 / 64)$ & $61.1(11 / 18)$ & $54.3(25 / 46)$ & 0.624 \\
\hline Histology & & & & 0.091 \\
\hline Thymoma AB & $15.6(10 / 64)$ & $22.2(4 / 18)$ & $13.0(6 / 46)$ & \\
\hline Thymoma B & $28.1(18 / 64)$ & $55.6(10 / 41)$ & $17.4(8 / 46)$ & \\
\hline Fusocelullar/mixed & $14.0(9 / 64)$ & $5.6(1 / 18)$ & $17.4(8 / 46)$ & \\
\hline Other & $12.5(8 / 64)$ & $5.6(1 / 18)$ & $15.2(7 / 46)$ & \\
\hline WHO stage & & & & 0.012 \\
\hline$A$ & $9.4(6 / 64)$ & $11.1(2 / 18)$ & $8.7(4 / 46)$ & \\
\hline$A B$ & $20.3(13 / 64)$ & $22.2(4 / 18)$ & $19.6(9 / 46)$ & \\
\hline $\mathrm{B}$ & $28.1(18 / 64)$ & $55.6(10 / 18)$ & $17.4(8 / 46)$ & \\
\hline III & $6.2(4 / 64)$ & $5.6(1 / 18)$ & $6.5(3 / 46)$ & \\
\hline IV & $31.2(20 / 64)$ & $33.3(6 / 18)$ & $30.4(14 / 46)$ & \\
\hline NE & $39.0(25 / 64)$ & $27.8(5 / 18)$ & $43.5(20 / 46)$ & \\
\hline Tumor size & & & & 0.993 \\
\hline Mean ( $\pm S D)$ & 67.05 (40.0) & $65.7(40.9)$ & $67.6(40.1)$ & \\
\hline
\end{tabular}

$\mathrm{SD}$, standard deviation.

$4(22 \%)$ patients in whom the diagnosis was made by other institutions before National Institute of Cancer admission.

\section{Discussion}

We studied 64 patients with thymoma and compared those with MG with those without MG, although clinical symptoms and autoimmune presentations were different, no difference was found in overall survival.

In our study, the age of onset, the frequency of the patients with TMG and the stage of diagnosis was consistent with the previous reports $(3,4,11,18)$. None of 
Table 2 MGFA classification of the 18 patients with thymoma associated myasthenia gravis

\begin{tabular}{lcc}
\hline MGFA & $\mathrm{n}[\%]$ & $\mathrm{P}$ \\
\hline $\mathrm{I}$ & $5[28]$ & \\
Ila & - & \\
Illb & $1[6]$ & \\
IIla & $3[17]$ & \\
IIIb & $7[39]$ & \\
IV & - & $<0.001$ \\
Not specified & $2[11]$ & \\
\hline
\end{tabular}

MGFA, Myasthenia Gravis Foundation of America.

Table 3 First-line treatment in patients with thymoma among patients with TMG and TnMG

\begin{tabular}{lcc}
\hline \multirow{2}{*}{$\begin{array}{l}\text { Treatment } \\
\text { modality }\end{array}$} & \multicolumn{2}{c}{ Thymoma } \\
\cline { 2 - 3 } & $\begin{array}{c}\text { With MG }(\mathrm{n}=18), \\
\%(\mathrm{n} / \mathrm{N})\end{array}$ & $\begin{array}{c}\text { Without MG }(\mathrm{n}=46), \\
\%(\mathrm{n} / \mathrm{N})\end{array}$ \\
\hline $\mathrm{Qx}$ & $38.9(7 / 18)$ & $17.4(8 / 46)$ \\
$\mathrm{RT}$ & $0(0)$ & $10.9(5 / 46)$ \\
Ch & $0(0)$ & $8.7(4 / 46)$ \\
Qx + Rt & $11.1(2 / 18)$ & $15.2(7 / 46)$ \\
Qx + Ch & $11.1(2 / 18)$ & $0(0)$ \\
Ch + RT & $5.6(1 / 18)$ & $34.8(16 / 46)$ \\
Qx + RT + Ch & $27.8(5 / 18)$ & $6.5(3 / 46)$ \\
Other & $5.6(1 / 18)$ & $4.3(2 / 46)$ \\
\hline
\end{tabular}

Qx, thymectomy; RT, radiotherapy; Ch, chemotherapy; TMG, thymoma with myasthenia gravis; TnMG, thymoma without myasthenia gravis.

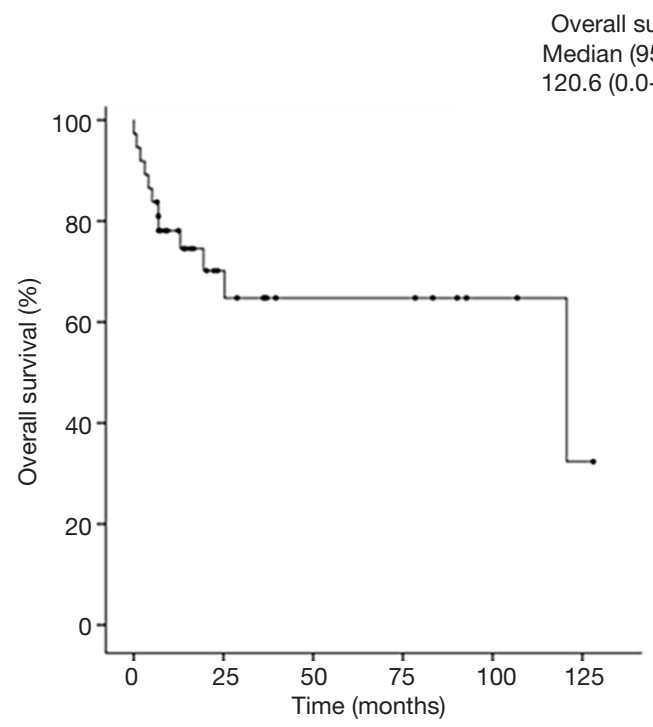

Table 4 Univariate analysis of the clinical factors associated with OS among patients

\begin{tabular}{|c|c|c|}
\hline Variable & Median, months $(95 \% \mathrm{Cl})$ & $\mathrm{P}$ \\
\hline Overall & $120.6(0.0-255.3)$ & - \\
\hline Gender & & 0.017 \\
\hline Female & $\mathrm{NR}(\mathrm{NR})$ & \\
\hline Male & $25.3(0.0-70.3)$ & \\
\hline Age, years & & 0.464 \\
\hline$<50$ & $120.6(25.3-N R)$ & \\
\hline$>50$ & $\mathrm{NR}(\mathrm{NR})$ & \\
\hline Histology & & 0.746 \\
\hline Thymoma & $\mathrm{NR}(\mathrm{NR})$ & \\
\hline Thymic carcinoma & $120.6(0.0-272.1)$ & \\
\hline WHO stage & & 0.247 \\
\hline$A, A B$ & $\mathrm{NR}(\mathrm{NR})$ & \\
\hline $\mathrm{B}, \mathrm{C}$ & $120.6(0.0-266.7)$ & \\
\hline Tumor size & & 0.245 \\
\hline$<5$ & NR (NR) & \\
\hline$>5$ & 120.6 (NR) & \\
\hline Myasthenia gravis & & 0.606 \\
\hline Absent & $120.6(0.0-264.9)$ & \\
\hline Present & NR (NR) & \\
\hline
\end{tabular}

NR, not reached.

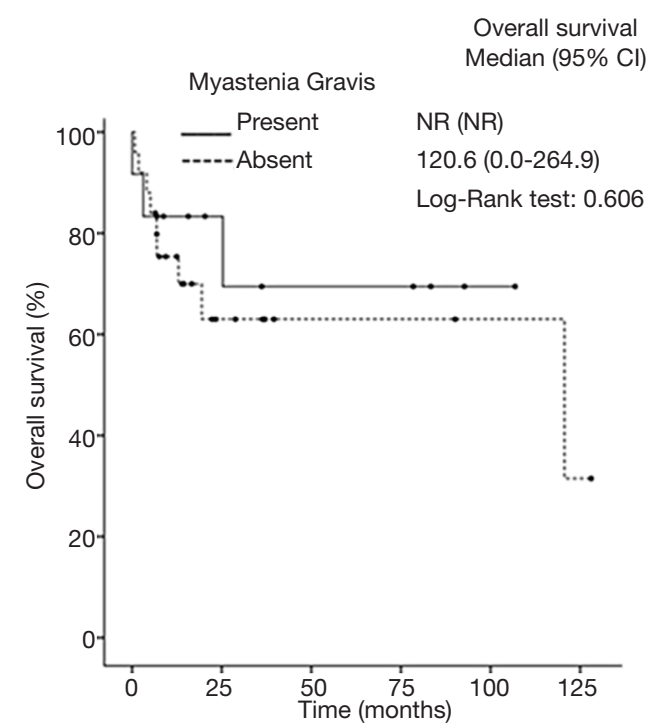

Figure 1 Kaplan-Meier curves for the OS of patients among all patients and according to the diagnosis or not of myasthenia gravis. 
Table 5 Clinical findings, metastases and AChR-Ab among patients with or without MG

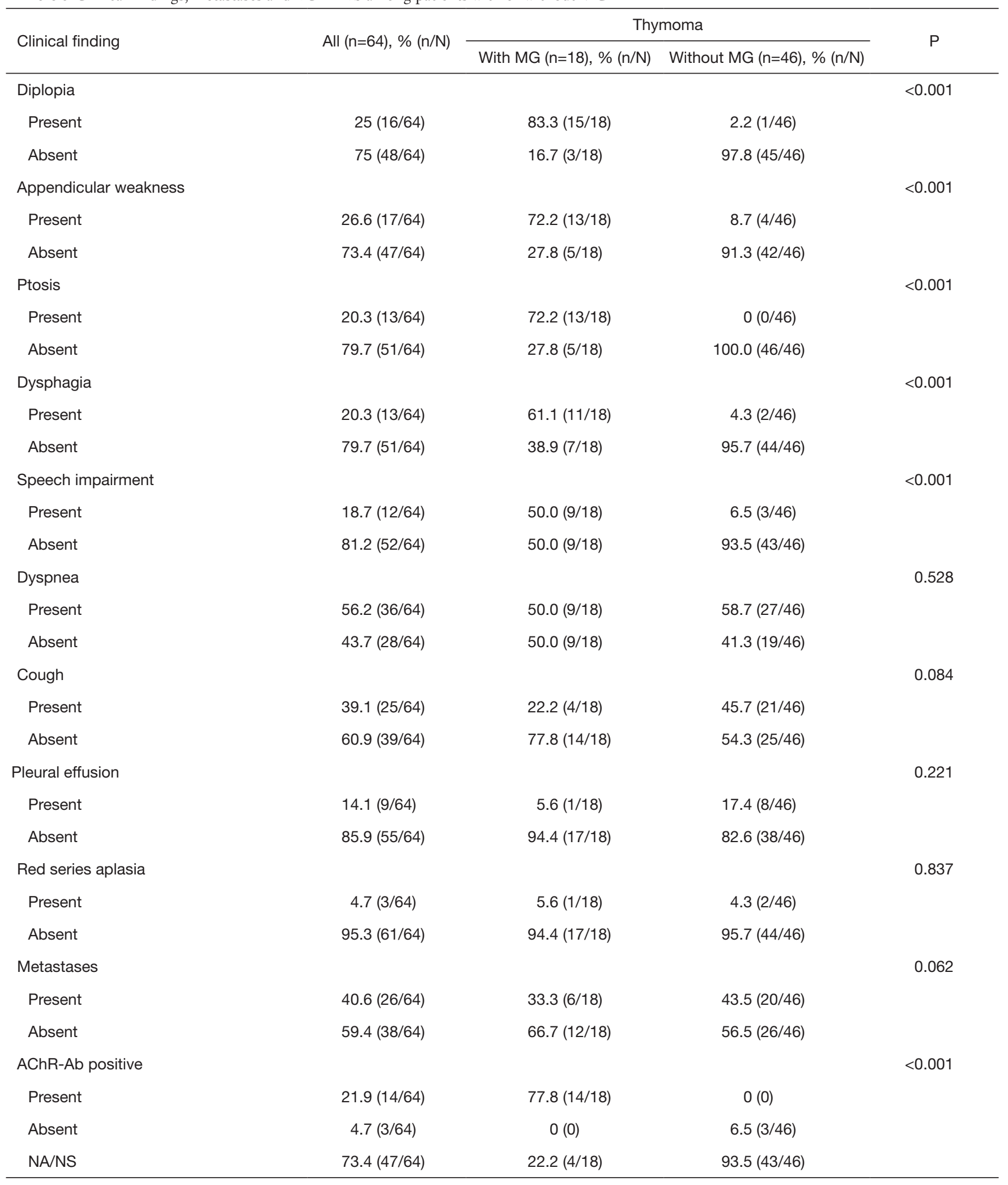

NA, not applicable; NS, not specified; AChR-Ab, acetylcholine receptor antibody. 
our patients from the TMG group were diagnosed with thymic carcinoma, although it has been described $(19,20)$. Nearly $90 \%$ of the patients with TMG had positive AChR$\mathrm{Ab}$, data that agrees with previous works and reports of the literature $(8,12,20)$. In two patients with TnMG, MG was suspected and was tested for AChR-Ab: one with diplopia and appendicular weakness and the second with dysphonia; both were negative and had thymic carcinoma. To our knowledge, just a small number of authors have reported TMG without AChR-Ab $(19,21)$. Pure red cell aplasia was found in $5 \%$ of all cases, similar to previous reports $(2,4)$. The Good's syndrome in patients with $\mathrm{T}$ has an estimated incidence of $5 \%(4,6)$, we found it in one patient with TMG and in one patient with TnMG. It has been described that patients with thymoma associated MG have a worse prognosis, and hence were likely to have a worse outcome $(12,22)$. As expected, our patients had several clinical manifestations of MG that had statistically significant differences, these manifestations were not remitted after thymoma treatment.

The prognostic value of $M G$ associated thymoma remains controversial in the different reports of the literature, some established the presence of MG as a good survival prognostic factor related to the onset of MG symptoms that leads a prompt approach and treatment (23-25); others reported that having MG or not, didn't show any statistical significance, same as our results (11,26-28); and finally, some studies reported even a worse prognosis in patients with MG $(17,29)$. This discrepancies are probably due to the wide range of clinical and pathogenic variants in MG patients, the present study has several limitations: the information was retrospectively acquired at a single institution, it includes only 64 patients and a referral bias should be considered, nevertheless, this report is valuable because in the best of our knowledge there is scarce information about MG and thymoma from Hispanic referral cancer centers; a study from 1991 had one of the largest population with MG and thymoma, and they reported a better overall survival in patients with MG compared with TnMG group (30). Since our database included patients that were detected years ago when the approach were less clearly and the patients were delayed in being referred to the appropriate specialist, most of our patients were in Masaoka stage IV, therefore, they had poor prognosis and high recurrence (13). According to the treatment 16 of TMG underwent thymectomy, one of them with partial resection; although there was a trend towards improved overall survival, this was not statistically significant in contrast to the reported by others (31).

Thymomas usually have no gender predilection (22), in our population we found an increased frequency in males, actually, after the univariate analysis we found that being male represents a prognostic factor associated with better overall survival $(\mathrm{P}=0.017)$.

Despite the low frequency of this disease, and given that there is still a lack of consensus on the impact of MG on overall survival, we encourage future studies to establish the optimal management and prognosis of thymoma patients and to find the best evidence.

\section{Conclusions}

In a group of Hispanic patients with thymoma, MG had a prevalence of $28 \%$, although we did not find statistically significant differences in overall survival; a larger multicentric study should be encouraged to have a better understanding of the prognostic implications of having $\mathrm{MG}$ in patients with thymoma. Moreover, it is recommended that all patients should be managed by a multidisciplinary team with experience in the management of TETs and with specific knowledge of the ongoing clinical trials.

\section{Acknowledgements}

None.

\section{Footnote}

Conflicts of Interest: The authors have no conflicts of interest to declare.

Ethical Statement: The study was reviewed and approved by our Institutional ethics and scientific committees (INCAN/ $\mathrm{CI} / 837 / 17)$.

\section{References}

1. Ettinger DS, Riely GJ, Akerley W, et al. Thymomas and thymic carcinomas: Clinical Practice Guidelines in Oncology. J Natl Compr Canc Netw 2013;11:562-76.

2. Scorsetti M, Leo F, Trama A, D 'angelillo R, et al. Thymoma and thymic carcinomas. Crit Rev Oncol Hematol 2016;99:332-50.

3. Kumar R. Myasthenia gravis and thymic neoplasms: A brief review. World J Clin Cases 2015;3:980-3.

4. Duwe BV, Sterman DH, Musani AI. Tumors of the 
mediastinum. Chest 2005;128:2893-909.

5. Marx A, Porubsky S, Belharazem D, et al. Thymoma related myasthenia gravis in humans and potential animal models. Exp Neurol 2015;270:55-65.

6. Kelleher P, Misbah SA. What is Good's syndrome? Immunological abnormalities in patients with thymoma. J Clin Pathol 2003;56:12-6.

7. Cufi P, Soussan P, Truffault F, et al. Thymoma-associated myasthenia gravis: On the search for a pathogen signature. J Autoimmun 2014;52:29-35.

8. Romi F, Hong Y, Gilhus NE. Pathophysiology and immunological profile of myasthenia gravis and its subgroups. Curr Opin Immunol 2017;49:9-13.

9. Fujii Y. The thymus, thymoma and myasthenia gravis. Surg Today 2013;43:461-6.

10. Berrih-Aknin S. Myasthenia Gravis: Paradox versus paradigm in autoimmunity. J Autoimmun 2014;52:1-28.

11. Aydemir B. The effect of myasthenia gravis as a prognostic factor in thymoma treatment. North Clin Istanb 2017;3:194-200.

12. Cacho Diaz B, Flores-Gavilán P, García-Ramos G, et al. Myasthenia gravis and its comorbidities. J Neurol Neurophysiol 2015;6:1-5.

13. Filosso PL, Evangelista A, Ruffini E, et al. Does myasthenia gravis influence overall survival and cumulative incidence of recurrence in thymoma patients? A Retrospective clinicopathological multicentre analysis on 797 patients. Lung Cancer 2015;88:338-43.

14. Masaoka A. Staging system of thymoma. J Thorac Oncol 2010;5:S304-12.

15. Wolfe GI, Kaminski HJ, Aban IB, et al. Randomized trial of thymectomy in myasthenia gravis. N Engl J Med 2016;375:511-22.

16. Marulli G, Rea F. Myasthenia gravis and thymectomy: many doubts and few certainties. Eur J Cardiothorac Surg 2015;48:46-7.

17. Zhang Z, Cui Y, Jia R, et al. Myasthenia gravis in patients with thymoma affects survival rate following extended thymectomy. Oncol Lett 2016;11:4177-82.

18. Safieddine N, Liu G, Cuningham K, et al. Prognostic factors for cure, recurrence and long-term survival after surgical resection of thymoma. J Thorac Oncol

Cite this article as: Cacho-Díaz B, Salmerón-Moreno K, Lorenzana-Mendoza NA, Texcocano J, Arrieta O. Myasthenia gravis as a prognostic marker in patients with thymoma. J Thorac Dis 2018;10(5):2842-2848. doi: 10.21037/jtd.2018.04.95
2014;9:1018-22.

19. Maggi L, Andreetta F, Antozzi C, et al. Two cases of thymoma-associated myasthenia gravis without antibodies to the acetylcholine receptor. Neuromuscul Disord 2008;18:678-80.

20. Vincent A, Bowen J, Newsom-Davis J, et al. Seronegative generalised myasthenia gravis: clinical features, antibodies, and their targets. Lancet Neurol 2003;2:99-106.

21. Richards J, Howard JF. Seronegative myasthenia gravis associated with malignant thymoma. Neuromuscul Disord 2017;27:417-8.

22. Gadalla SM, Rajan A, Pfeiffer R, et al. A population-based assessment of mortality and morbidity patterns among patients with thymoma. Int J Cancer 2011;128:2688-94.

23. Wang F, Pang L, Fu J, et al. Postoperative survival for patients with thymoma complicating myasthenia gravispreliminary retrospective results of the ChART database. J Thorac Dis 2016;8:711-7.

24. Akaishi T, Suzuki Y, Imai T, et al. Response to treatment of myasthenia gravis according to clinical subtype. BMC Neurol 2016;16:225.

25. Li W, Miao Z, Liu X, et al. Thymic carcinoma patients with myasthenia gravis exhibit better prognoses. Int J Clin Oncol 2016;21:75-80.

26. Kondo K, Monden Y. Thymoma and myasthenia gravis: a clinical study of 1,089 patients from Japan. Ann Thorac Surg 2005;79:219-24.

27. Lee WS, Heo DS, Bang YJ, et al. Prognostic factors of patients with thymoma. Korean J Intern Med 1996;11:40-9.

28. Yu L, Zhang XJ, Ma S, et al. Different characteristics of thymomas with and without myasthenia gravis. Ann Surg Oncol 2012;19:94-8.

29. Li L, Wang Y, Peng T, et al. Metformin restores crizotinib sensitivity in crizotinib-resistant human lung cancer cells through inhibition of IGF1-R signaling pathway. Oncotarget 2016;7:34442-52.

30. Maggi G, Casadio C, Cavallo A, et al. Thymoma: results of 241 operated cases. Ann Thorac Surg 1991;51:152-6.

31. Mussi A, Lucchi M, Murri L, et al. Extended thymectomy in myasthenia gravis: a team-work of neurologist, thoracic surgeon and anaesthesist may improve the outcome. Eur J Cardiothorac Surg 2001;19:570-5. 